Supporting Information

\title{
Novel Fluorescence Method for Determination of Spatial Interparticle Distance in Polymer Nanocomposites
}

Zekun Zhang, ${ }^{\dagger, \#}$ Zemin Feng, ${ }^{\dagger, \#}$ Rui Tian, ${ }^{\dagger, *}$ Kaitao Li, ${ }^{\dagger}$ Yanjun Lin, ${ }^{\dagger}$ Chao Lu, ${ }^{\dagger}, *$ Shuhua Wang, ${ }^{\ddagger}$ and Xingjie Xue

'State Key Laboratory of Chemical Resource Engineering, Beijing University of Chemical Technology, Beijing 100029, China

${ }^{\sharp}$ Kemai Chemical Co., Ltd., Tianjin 300270, China

${ }^{\#}$ Z.K. Zhang and Z.M. Feng contributed equally to this work

*E-mail:tianrui@mail.buct.edu.cn

*E-mail: luchao@mail.buct.edu.cn 


\section{Contents}

Figure S1. (A) XRD pattern and (B) SEM image of layered double hydroxides (LDHs).

Figure S2. Infrared transmittance of LDHs in the range of $600-4000 \mathrm{~cm}^{-1}$.

Figure S3. Fluorescence excitation and emission spectra of 3-(10-phenyl-9-anthracenyl) phenyl boronic acid (DPBA) in alkaline buffer solution, and the inset shows the molecular structure of DPBA.

Figure S4. Fluorescent emission spectra of low-density polyethylene (LDPE) films before (black line) and after (blue line) fluorescent labeling by DPBA probe.

Figure S5. Two-dimensional fluorescence images for $2.0 \mathrm{wt} \% \mathrm{LDHs} / \mathrm{LDPE}$ film at different depths.

Figure S6. MATLAB program for determination of spatial interparticle distance (ID).

Table S1. The spatial ID data for LDHs/LDPE PNCs with different contents of LDHs. 

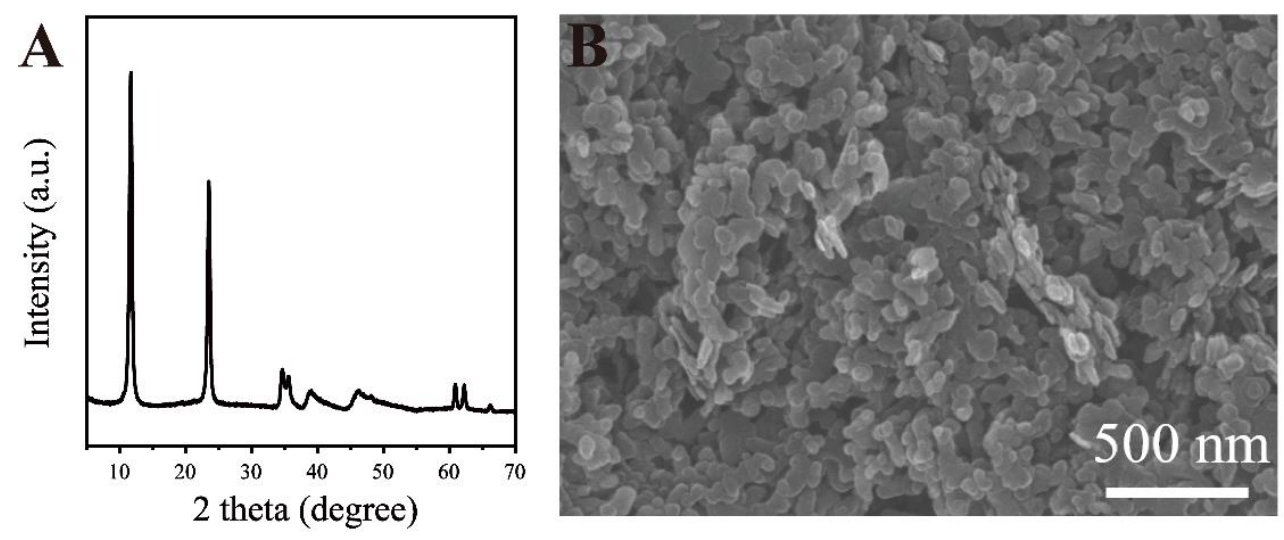

Figure S1. (A) XRD pattern and (B) SEM image of layered double hydroxides (LDHs). 


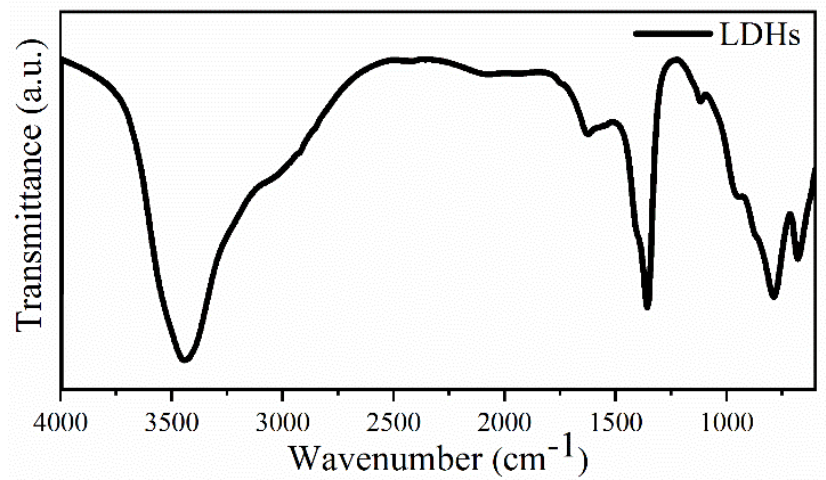

Figure S2. Infrared transmittance of LDHs in the range of $600-4000 \mathrm{~cm}^{-1}$. 


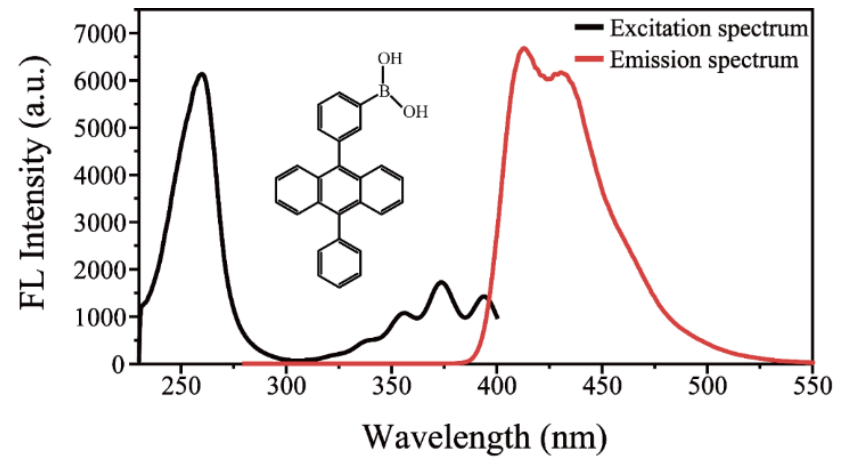

Figure S3. Fluorescence excitation and emission spectra of 3-(10-phenyl-9-anthracenyl) phenyl boronic acid (DPBA) in alkaline buffer solution, and the inset shows the molecular structure of DPBA. 


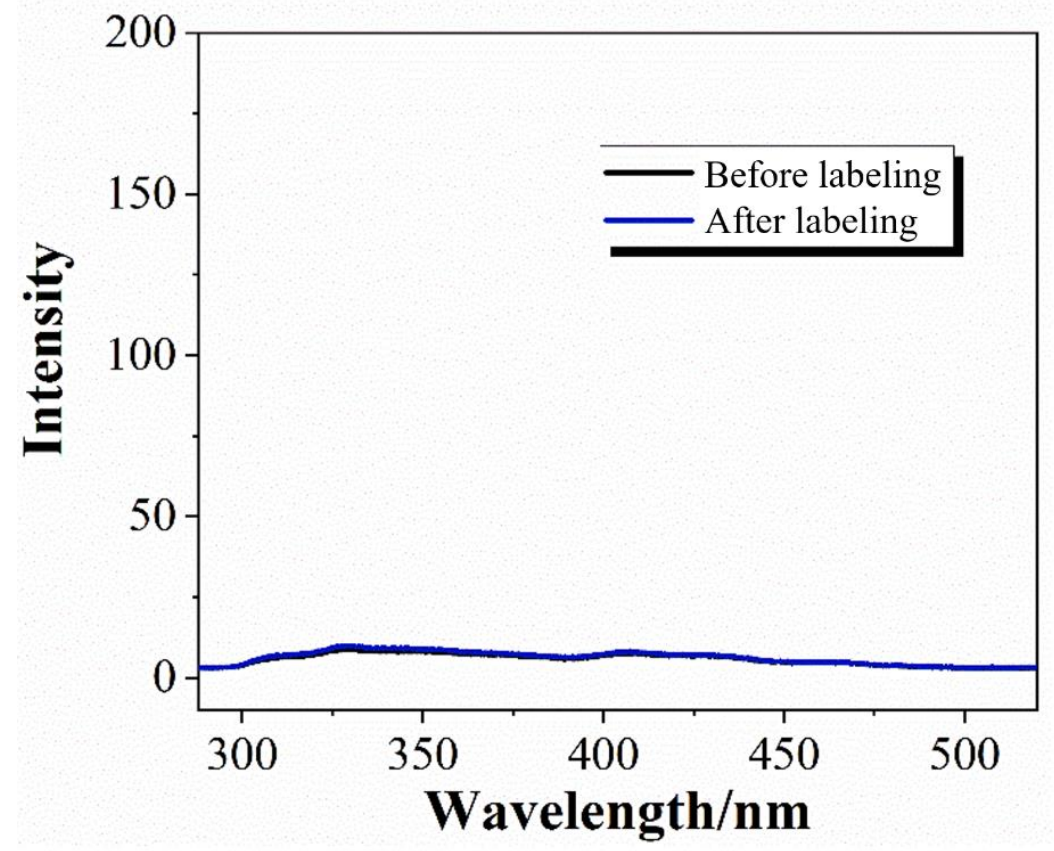

Figure S4. Fluorescent emission spectra of low-density polyethylene (LDPE) films before (black line) and after (blue line) fluorescent labeling by DPBA probe. 


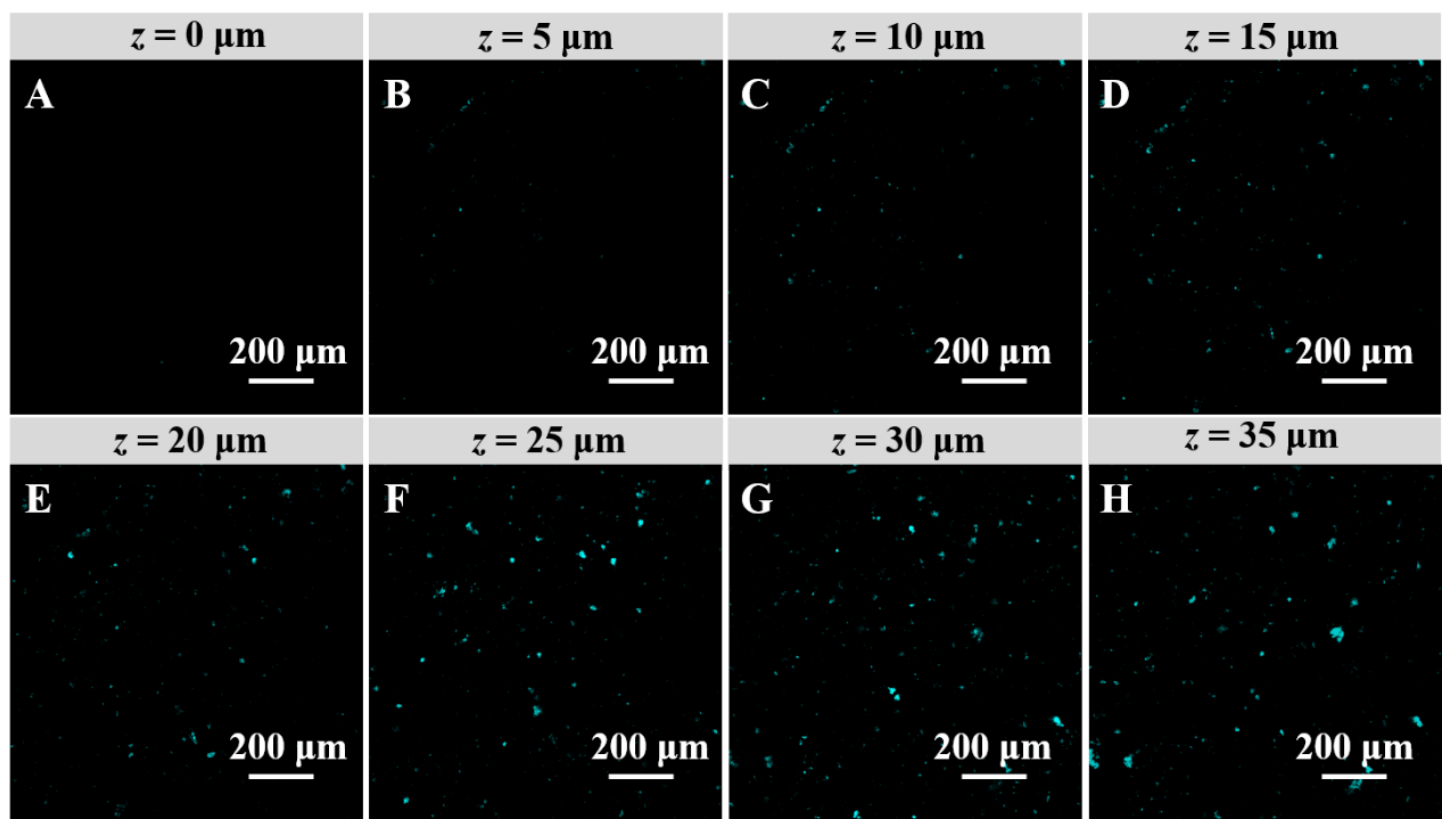

Figure S5. Two-dimensional fluorescence images for $2.0 \mathrm{wt} \% \mathrm{LDHs} / \mathrm{LDPE}$ film at different depths. 
MATLAB script for calculation

data $=$ xlsread('Data.xlsx');

distance $=$ squareform(pdist(data));

ind $=$ find $($ eye $($ size $($ distance, 1$)))$;

distance(ind)=inf;

Min_distance $=\min ($ distance $)$;

figure;

hist(Min_distance);

xlabel('nearest interparticle distance $(\mu \mathrm{m})$ ')

ylabel('point')

Figure S6. MATLAB program for determination of spatial interparticle distance (ID). 
Table S1. The spatial ID data for LDHs/LDPE PNCs with different contents of LDHs.

\begin{tabular}{ccccc}
\hline Content of LDH $(\mathbf{w t} \%)$ & ID-1 $(\boldsymbol{\mu m})$ & ID-2 $(\boldsymbol{\mu m})$ & ID-3 $(\boldsymbol{\mu m})$ & Average $(\boldsymbol{\mu m})$ \\
\hline 0.2 & 23.4 & 20.4 & 22.6 & 22.2 \\
0.4 & 16.2 & 16.7 & 15.8 & 16.2 \\
0.6 & 14.6 & 13.4 & 13.0 & 13.7 \\
0.8 & 11.6 & 11.4 & 11.5 & 11.5 \\
1.0 & 10.1 & 9.8 & 9.7 & 9.9 \\
1.2 & 9.1 & 9.0 & 9.3 & 9.1 \\
1.4 & 7.7 & 7.8 & 7.8 & 7.8 \\
1.6 & 7.9 & 8.1 & 8.0 & 8.0 \\
1.8 & 8.3 & 8.5 & 8.2 & 8.3 \\
2.0 & 10.8 & 10.5 & 10.4 & 10.5 \\
\hline
\end{tabular}

